\title{
MINIREVIEW
}

\section{Hepatitis B virus vaccine breakthrough infection: surveillance of $S$ gene mutants of $\mathrm{HBV}$}

\author{
Y. QIN ${ }^{1}$, P. $\operatorname{LIAO}^{2 *}$ \\ ${ }^{1}$ Department of Laboratory Medicine, Yongchuan Hospital of Chongqing Medical University, No.439, Xuanhua West Road, \\ Yongchuan, Chongqing, 402160, P. R. China; ${ }^{2}$ Department of Laboratory Medicine, Chongqing General Hospital, No. 104, Pipashan \\ Street, Yuzhong District, Chongqing, 400000, P. R. China
}

Received June 7, 2017; accepted August 22, 2017

\begin{abstract}
Summary. - Hepatitis B (HB) is a worldwide public health problem, closely related with liver cirrhosis and hepatocellular carcinoma (HCC). The implementation of universal hepatitis B virus (HBV) vaccination programs has led to significant reduction in incidence of acute and chronic HB, liver cirrhosis and HCC. However, this success is now being threatened by the discovery of $\mathrm{HBV}$ vaccine breakthrough infection caused by the $\mathrm{S}$ gene mutants of $\mathrm{HBV}$, high maternal viral load and virus-induced immunosuppression. An alteration in the antigenicity and immunogenicity of hepatitis $B$ surface antigen (HBsAg) due to $S$ gene mutations may compromise detection of HBsAg (diagnosis-escape mutants), treatment with hepatitis B-specific immunoglobulin (HBIG), and even cause infections in individuals who are antihepatitis B surface antigen (anti-HBs) antibody-positive after immunization (vaccine-escape mutants). By surveilling for S gene mutants of HBV among vaccinated population, we will have a better understanding of the mechanism of HBV vaccine breakthrough infection; potentially providing new ideas for designing better diagnostic assays and effective vaccines for prevention and treatment of HBV. This review attempts to briefly summarize the status and role of S gene mutations, B-cell epitopes and T-cell epitope mutants, and surveillance of mutant HBV variants in a hospital setting.
\end{abstract}

Keywords: HBV vaccine breakthrough infection; S gene; mutants; surveillance

\section{Contents}

1. Introduction

2. Definition of HBV vaccine breakthrough infection

3. Causes of vaccine breakthrough infection

4. S gene mutants of $\mathrm{HBV}$

5. Mutations in surface protein B-cell epitopes

6. Mutations in surface protein T-cell epitopes

*Corresponding author. E-mail: PuLiao2002@163.com; phone: +13628358003 .

Abbreviations: anti-HBc $=$ hepatitis $\mathrm{B}$ core antigen; anti$\mathrm{HBs}=$ hepatitis $\mathrm{B}$ surface antigen; $\mathrm{CTL}=$ cytotoxic $\mathrm{CD} 8+$ lymphocyte; $\mathrm{HB}=$ hepatitis $\mathrm{B}$; HBIG = hepatitis B-specific immunoglobulin; $\mathrm{HBsAg}=$ hepatitis $\mathrm{B}$ surface antigen; $\mathrm{HBV}=$ hepatitis $\mathrm{B}$ virus; $\mathrm{HCC}=$ hepatocellular carcinoma; $\mathrm{MHC}=$ major histocompatibility complex; MHR = major hydrophilic region
7. S gene mutations in patients with $\mathrm{HBV}$ vaccine breakthrough infection

8. Surveillance for S gene mutants of HBV vaccine breakthrough infection patients

9. Conclusion

\section{Introduction}

Chronic HBV infection continues to be a major public health concern worldwide despite the availability of an effective vaccine and potent antiviral treatments (Beasley, 2009). A global systematic review showed that in 2010, about 248 million individuals in the general population were chronically infected with HBV worldwide. China has been classified as the higher intermediate-endemic country because 
HBsAg-positive population of China is 74 million, i.e., 5.49\% (5.47-5.50) of the total Chinese population (Schweitzer et al., 2015), which is lower than the epidemiological data of China in 2006 (93 million HBsAg-positive carriers). HBV is mainly transmitted via mother-to-infant and household contact in endemic countries (Edmunds et al., 1996), and through intravenous drug usage and high-risk sexual behavior in more industrialized countries (Mast et al., 2006). However, the patterns of HBV infection in vaccinated population are unknown. HBV, has a unique life cycle including the activity of error-prone reverse transcriptase, high virion production per day and the ease with which it can mutate, resulting in different genetic variants. $S$ gene mutants have important clinical significance in vaccinated population as they may infect vaccinated individuals because anti-HBs antibodies induced by the current vaccine may not recognize changes in HBsAg caused by $\mathrm{S}$ gene mutations. Thus, $S$ mutant breakthrough infections may occur in vaccinated people, putting the whole population at risk (Hudu et al., 2015). There are significant differences in the distribution of S gene mutations in various genotypes (Ma et al., 2012). Universal $\mathrm{HBV}$ vaccination programs has been implemented for all neonates in China since 1992, effectively preventing the occurrence of chronic liver disease and HCC (Li et al., 2004) Nevertheless, HBV vaccine breakthrough infection and occult hepatitis B infection (OBI) have been found in vaccinated subjects and blood donors, which may be associated with $S$ gene mutants of HBV. Thus, extensive molecular characterization of vaccine-escaped hepatitis B strains needs to be performed.

For this review, we identified studies by searching PubMed for articles published from January 2006 to December 2016 using the keywords "HBV vaccine breakthrough infection", "HBV" and "Vaccine-Escape Mutant". Additional papers were identified by a manual search of references from key articles.

\section{Definition of HBV vaccine breakthrough infection}

HBV vaccine breakthrough infection is an infection caused by $\mathrm{HBV}$ in an individual with validated history of full primary hepatitis $\mathrm{B}$ vaccination and who is $\mathrm{HBsAg-}$, antihepatitis B core antigen (anti-HBc)- or HBV DNA-positive regardless of the serostatus of anti-HBs. There is no standard definition of HBV vaccine breakthrough infection. In an international meeting organized by the Viral Hepatitis Prevention Board in Milan, Italy, participants expressed the need for further clarification and standardization of the definition of breakthrough infection, since the term covered situations ranging from infection with $\mathrm{HBV}$ in a person unresponsive to primary hepatitis $B$ vaccination to infection because of an unknown vaccine failure (FitzSimons et al., 2013). The prevalence of S gene mutants varies among HBV genotypes. There was a prevalence of 5.5\% for hepatitis B-escaped mutants among blood donors and vaccinated undergraduates, with the most common mutation being found at amino acid position 16 (G16L) of S gene (Hudu et al., 2015). The Chinese prevalence is $7.7 \%$; among them $1 \%$ were found to be HBsAg-positive and $6.7 \%$ were $\mathrm{HBsAg}$-negative and anti-HBc-positive (Zhu et al., 2011).

\section{Causes of vaccine breakthrough infection}

The main reasons for the hepatitis B vaccine breakthrough infection are not very clear as very little research has been carried out in this area. Studies have shown that this may be associated with the emergence of $S$ gene mutants, high maternal viral load, immunosuppression, and intrauterine infection (Chang, 2010). A ten-year surveillance study of breakthrough HBV infections in vaccinated people in Italy showed that out of seven correctly vaccinated and HBV DNA-positive subjects, four cases were attributable to a lack of immune response and other three to viral mutants able to evade the immune response. The main risk factors of infection include household HBsAg-positive, intravenous drug use, and hemodialysis (FitzSimons et al., 2013). Besides the opinions above, there is also opinion that the HBV vaccine breakthrough infection is mainly caused by the wild type genotype $\mathrm{E}$ strain and that immune escape mutants are uncommon (Mendy et al., 2008). However, HBV mutants may play a role in establishing infection later in life when anti-HBs antibody concentration has begun to decline. At the same time, a study in Taiwan showed that most HBV breakthrough infections are due to maternal transmission and immunized children born to genotype $\mathrm{C}$ mothers may have a higher rate of breakthrough infections than those born to genotype B mothers (Wen et al., 2011). Another research found that although non-responders to $\mathrm{HBV}$ vaccination (anti-HBs $<10 \mathrm{mUI} / \mathrm{ml}$ in serum) have a suboptimal B cell response, nonetheless it may be enough to protect against clinically significant breakthrough hepatitis B infection (Valats et al., 2010).

\section{4. $S$ gene mutants of $\mathrm{HBV}$}

The HBV genome has four open reading frames (ORFs) named X, C, P and S. Surface protein, including T-cell and B-cell antigenic epitopes (Table 1), is coded by S ORF, which is the major HBV antigen that mediates virus attachment and entry and determines virus genotype. HBV mutants may be selected under immune pressure or therapy with antiviral drugs or naturally occur and accumulate during chronic infection. Immune-escape mutants that emerge 
Table 1. The epitopes and corresponding mutant sites of $\mathrm{HBsAg}$

\begin{tabular}{|c|c|c|}
\hline HBsAg epitopes & Amino acid position & Mutations \\
\hline \multirow[t]{2}{*}{ B-cell } & $\begin{array}{l}\text { 100-160 (Gerlich et al., 2006) } \\
\text { (exclusion124-147) }\end{array}$ & $\begin{array}{l}\text { P120T/S/Q, T123A/N (Hudu et al., 2015); S114T/F (Ziaee et al., 2016; Mizukoshi et al., } \\
\text { 2004); K160R, I110L, S113T (Ziaee et al., 2016); V106A, T118V (Larralde et al., 2013). }\end{array}$ \\
\hline & 124-147(“a” determinant region) & $\begin{array}{l}\text { G145A/R (Ma et al., 2012; Lin et al., 2013; Ziaee et al., 2016); D144A/E (Ma et al., } \\
\text { 2012; Lin et al., 2013; Ye et al., 2015); N131T (Ma et al., 2012; Lin et al., 2013; Ye et } \\
\text { al., 2015); T126S/I, Q129H/R, S143L, M133L (Honorati et al., 1997; Lin et al., 2013); } \\
\text { G130N, S140T (Ma et al., 2012). }\end{array}$ \\
\hline \multirow[t]{4}{*}{ T-helper } & 19-28 (Larralde et al., 2013) & F20S, T23A (Larralde et al., 2013). \\
\hline & 80-98 (Bauer et al., 2002) & F85C (Lin et al., 2013). \\
\hline & $\begin{array}{l}\text { 186-197 (Mancini-Bourgine et } \\
\text { al., 2006) }\end{array}$ & I194V (Lin et al., 2013); L186P, I195M (Ziaee et al., 2016). \\
\hline & 215-223 (Bauer et al., 2002) & L216*, W223*(Ziaee et al., 2016). \\
\hline \multirow[t]{4}{*}{ CTL } & 28-51(Tai et al., 1997) & S45T, V47T, L49P, L42S (Lin et al., 2013); P29S, S34L (Larralde et al., 2013). \\
\hline & 171-179 (Shahmoradi et al., 2012) & S173F(Ziaee et al., 2016); S171F, S174N (Ye et al., 2015). \\
\hline & $\begin{array}{l}\text { 175-184 (Mancini-Bourgine et } \\
\text { al., 2006) }\end{array}$ & Q181R (Ye et al., 2015). \\
\hline & $\begin{array}{l}\text { 206-215 (Mancini-Bourgine et } \\
\text { al., 2006) }\end{array}$ & S207N (Lin et al., 2013). \\
\hline $\begin{array}{l}\text { TH/CTL } \\
\text { Inter-epitopes }\end{array}$ & - & $\begin{array}{l}\text { Y200H/F (Ziaee et al., 2016; Lin et al., 2013); E164G/V (Ziaee et al., 2016; Bauer et al., } \\
\text { 2002); P56Q, T57N, N59S, S64C (Lin et al., 2013); R78Q (Ziaee et al., 2016). }\end{array}$ \\
\hline
\end{tabular}

under active and/or passive vaccination and are responsible for HBV vaccine breakthrough infection are of particular clinical relevance (Gerlich, 2006).

Mutation in the surface protein is one of the most powerful viral strategies for escaping recognition by both the B- and T-cell-mediated immune responses. As a structural protein, HBsAg serves as an immune target. Mutations in B-cell and T-cell epitopes are likely to significantly alter the immunological characteristics of HBsAg, rendering MHC and MHC class II-restricted T-helper (TH) cells to recognize B-cell and T-cell, restrictively. In addition, these mutations also result in decreased binding affinity by MHC class I-mediated presentation of modified oligopeptides on the cell surface of hepatocytes and enable HBV to escape immune surveillance and immune clearance (Bauer et al., 2002; Mizukoshi et al., 2004).

\section{Mutations in surface protein B-cell epitopes}

B-cell epitopes are clustered within the major hydrophilic region (MHR; aa 100-160) (Honorati et al., 1997). The region of amino acids 124-149 within MHR, known as the "a" determinant gene, is the major region for mutation. The "a" determinant region of HBsAg is the main target for vaccine development, including antibody production, which can protect against infection by all HBV genotypes (from A to J). Mutations in the surface protein, a result of amino acid deletions or substitutions, may lead to the conformational change of epitopes affecting the antigenicity and immunogenicity particularly in the "a" determinant gene, resulting in immune escape (Wu et al., 2010). Thus, the mutant virus cannot be identified and removed by the immune system of the host and can infect both vaccinated and unvaccinated individuals. Escape mutations in surface protein B-cell epitopes are reported widely worldwide. Generally, mutations were more likely to be discovered at amino acid positions 120,123 , $126,129,130,133,134,137,139,140$, and 143-145, and a subject generally exhibits multiple mutations. Most of the mutations were located at the "a" determinant region (Ma et al., 2012; Lin et al., 2013; Ye et al., 2015; Ziaee et al., 2016), indicating the region is more likely to be affected by immune selection. G145A appears to be the most common escape mutation induced by anti-HBs immune pressure (Lin et al., 2013; Ma et al., 2012; Ziaee et al., 2016). A study found that eight important mutations associated with diagnostic failure are P120T, T126S, Q129H, G130N, S143L, D144A, and G145A/R; whereas the mutations related to vaccine escape and HBIG therapy failure were located mainly at positions $120,126,129,130,133,134,137,140,143,144$ and 145 (Ma et al., 2012). Further, there are differences in the position of mutations among vaccinated and unvaccinated subjects. Hsu et al. found that vaccinated subjects had a tendency of higher mutation rates in "a" determinant of HBsAg than unvaccinated subjects (Hsu et al., 2015). Mutations occur more frequently in the second loop (aa124-137) than in the first loop (aa139-149) of the "a" determinant region (Hsu et al., 2004; Michler et al., 2014). 


\section{Mutations in surface protein T-cell epitopes}

Mutations in T-cell epitopes may in theory influence the $\mathrm{HBs} \mathrm{Ab}$ profile through altered interaction between CD4+ TH cells and B-cells (Cooreman et al., 2001). Suitable T-cell response is a prerequisite to producing enough antibodies after $\mathrm{HBV}$ infection and vaccination. The humoral response to HBsAg depends on T cells, since four regions within $\mathrm{HBsAg}$, which contain epitopes for MHC class II-restricted CD4+ T-cells have been reported (Larralde et al., 2013; ManciniBourgine et al., 2006). Thus, mutations in TH-cell epitopes will affect humoral response and anti-HBs antibody production (Shi et al., 2014). Mutants of HBsAg also have the potential to escape cellular immune response of individuals vaccinated against $\mathrm{HBV}$ due to the loss of immune recognition of $\mathrm{TH}-$ cell epitopes. Bauer et al. (2002) had a study on 30 vaccinated volunteers to test $\mathrm{T}$-cell reactivity toward 23 reported mutants at T-cell epitopes of HBsAg. These mutations mainly occurred in P1 (aa16-33) and P4 (aa213-216), and six of them resulted in complete or significant loss of T-cell reactivity because these mutated epitopes could not activate T cells. In addition, it has been experimentally proved that adaptive immune response mediated by MHC class I-restricted cytotoxic CD8+ lymphocytes (CTLs) is necessary for controlling HBV infection (Zhang et al., 2012). In H-2b mice, even small changes in amino acid residues within two different CTL epitopes (aa208-215, aa190-197) completely eliminated the immunogenicity of each epitope (Schirmbeck et al., 2003). Ye et al. (2015) showed that there were three new mutations (S171F, S174N, Q181R) at the position of the MHC class -restricted CTL epitopes of HBsAg in patients with chronic hepatitis B, suggesting that these mutations might contribute to chronic infections. This may be because mutations in CTL epitopes can evade cellular immunity and contribute to persistency (Franzese et al., 2005; Zuckerman AJ, 2000). In summary, a number of mutations upstream and downstream of MHR were found to be located within the known $\mathrm{T}$ cell epitopes of HBsAg. These mutations lead to amino acid changes, which may contribute to T-cell non-responsiveness or an inappropriate $\mathrm{T}$-cell response, and are potentially responsible for vaccine breakthrough infection and HBsAg undetectability (Ma et al., 2012; Lin et al., 2013; Ye et al., 2015; Ziaee et al., 2016).

\section{7. $S$ gene mutations in patients with $\mathrm{HBV}$ vaccine breakthrough infection}

The mechanisms by which mutated virus causes HBV vaccine breakthrough infection include: 1) altered conformational structure and/or T-cell epitope structure allowing mutated virus to evade recognition and clearance by the immune system; 2) altered antigenicity of HBsAg through changes in hydrophobicity profile and putative glycosylation site, which consequently decrease or abolish affinity for anti-HBs, offering lower protection from vaccination (Luongo et al., 2015) and permitting infection with HBV isolates of different genotypes or subtypes in patients with a normal anti-HBs response (Wu et al., 2012); and 3) the accumulation of aa substitutions because of mutations in and around the "a" determinant region may change immunogenicity of $\mathrm{HBsAg}$, producing less effective neutralizing anti-HBs response and enabling the mutated virus evade clearance and survive long terms (Kfoury et al., 2001). S gene mutations of HBV can occur in vaccinated population with fulminant, acute and chronic HB. Similar HBsAg escape mutations in 2nd loop of the "a" determinant (S143L; S143 M) were observed in two virus strains causing fulminant hepatitis, but no such mutations were detected in asymptomatic and chronic hepatitis B patients (T. Michler et al., 2014). Luongo et al. also reported a male patient with high seroprotective anti-HBs titer (>200 IU/l) and acute HB with three mutations (M125T, T127P and Q129H) in the virus strain (Kfoury et al., 2001). S gene mutants are the dominant HBV variants in immunized population with fulminant and acute HB (Hsu et al., 1997), because these mutations allow the virus to escape neutralization by antibodies and inefficient CTL-mediated control. HBsAg escape mutations have mostly been described in the context of chronic infections. There was a research that found five of 8 completely vaccinated individuals, who were seropositive for HBV DNA, carried variants with mutations in the $S$ gene. The localization of mutations spread over the entire surface protein, including S114T, T131N, G145A, E164V, R78Q, L173F, L186P, I195M, Y200H, L216*, W223 (Lai et al., 2012). Other researchers have also found some meaningful mutations, such as P120T, T126S, Q129H, S143L/M, D144A, G145A/R and more (Ma et al., 2012; Lin et al., 2013; Ye et al., 2015; Ziaee et al., 2016). More often, there are more than one concurrent mutations of HBsAg and they may have cumulative effects on the conformation of HBsAg (Wu et al., 2010). In recent studies, significant differences were found in the distribution of escape mutants in various genotypes (Ma et al., 2012). Genotypes display a certain geographical distribution and have different clinical significance in diagnosis of mutated virus, vaccine design and immunoglobulin treatment (Chotiyaputta et al., 2009). Therefore, it is meaningful to systematically study the frequency of HBsAg mutations in different geographical areas and different genotypes. Since HBV genotypes A-D are more prone to harbor escape mutants, they should be monitored more closely (Ma et al., 2012).

\section{Surveillance for $\mathrm{S}$ gene mutants of $\mathrm{HBV}$ vaccine breakthrough infection patients}

Even though effective vaccine and potent antiviral treatments are available to control HBV infections, there are still 
problems hindering full control of $\mathrm{HB}$ and to completely clear HBV and HBV escape mutants. In the last 20 years, numerous studies have shown that $\mathrm{S}$ gene mutations have impact on the progress of the disease, the reliability of diagnostic method and success of vaccination and antiviral therapy. Furthermore, anti-HBs immune pressure-induced HBV escape mutants in vaccinated people lead to the spread of mutated HBV. Besides the vaccinated population, the surveillance of $S$ gene mutants is also very important to the children whose households have HBsAg carriers, drugresistant patients, or other high-risk groups, such as blood donors, intravenous drug users, and adolescents with sexual promiscuity. Thus, surveillance of the molecular epidemiology of hepatitis B mutants is very important. We recommend the following roadmap for surveillance of hepatitis B mutants in a hospital setting (Fig. 1). On encountering a subject who has completed the primary vaccination, HBsAg-, anti-HBcand HBV DNA-positive should be checked no matter his or her anti-HBs status to understand whether breakthrough infection occurs. Then, sequencing of the surface gene as well as genotyping should be done to clarify whether $S$ gene mutant is present in those, whose HBV DNA is positive.

\section{Conclusion}

Numerous studies have confirmed that the implementation of universal $\mathrm{HBV}$ vaccination programs has significantly reduced HBsAg carriage and infection rates worldwide. Nevertheless, an increased seroprevalence and a high percentage of HBsAg mutants, especially G145R/A and T126S have been found in individuals with $\mathrm{HBV}$ vaccine breakthrough infection. Although present vaccines are capable of inducing immunity against the mutants; long-term surveillance and more research on $S$ gene mutants is required for identification of additional mutant variants with significant effect on B and T-cell levels, their frequencies and geographical distribution. The information obtained from these studies would provide new insight into the pathogenesis of HBV vaccine breakthrough infection and occult hepatitis B infection to reduce

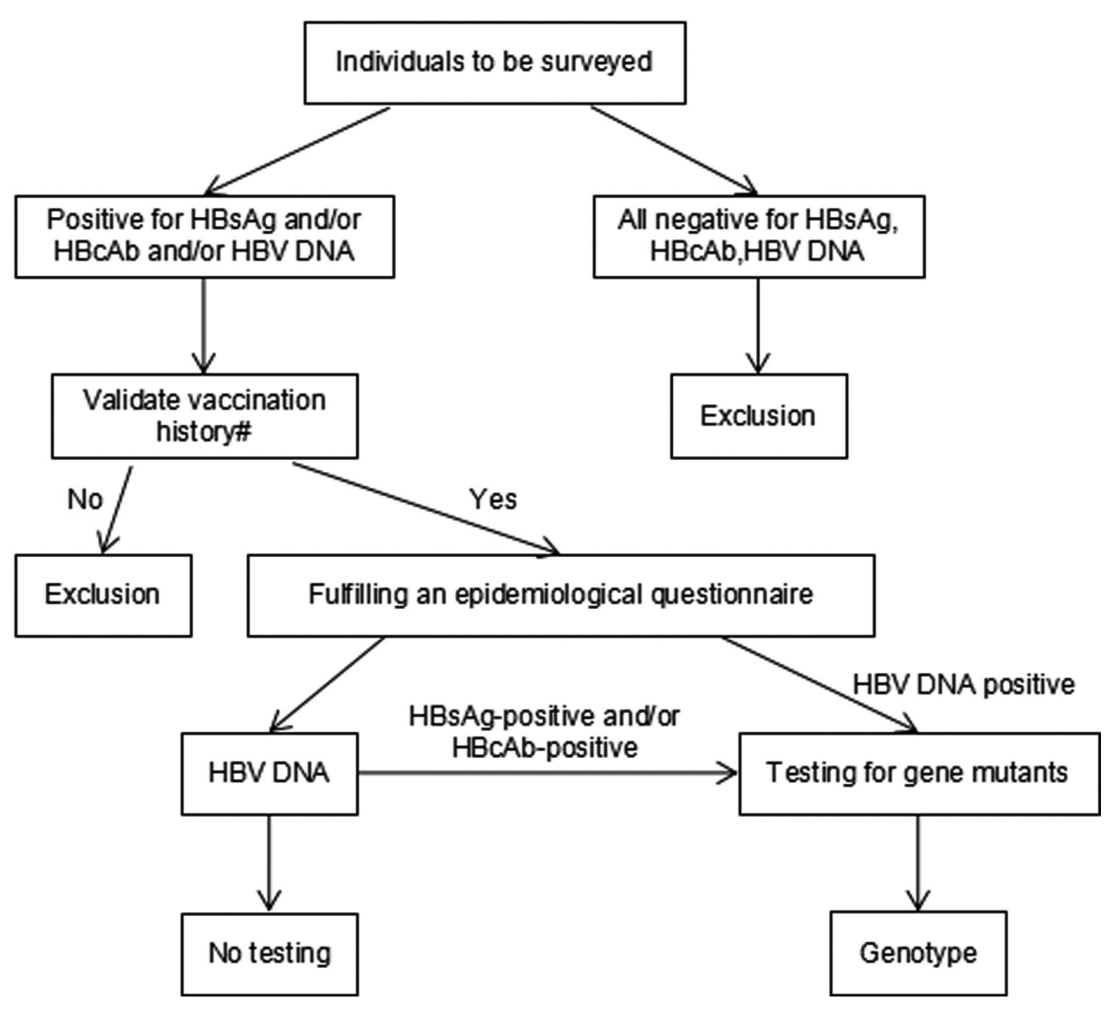

Fig. 1

The flowchart of HBV $S$ gene mutation surveillance

First to determine research objects who are seropositive for HBsAg or anti-HBc or HBV DNA, regardless of their serostatus for anti-HBs. And a subject should fulfill an epidemiological questionnaire (including basic information, $\mathrm{HB}$ vaccination history, risk factors and so on). We will check the serum for HBV DNA if one has accomplished vaccination. Serum found to be positive for HBV DNA are then processed for sequencing to identify $S$ gene mutation and genotype. \#Validate vaccination history: Firstly, there are three separate doses of vaccination following the immunization schedules. Secondly, HBV infection occurred after completing the vaccination. 
HBsAg carrier rate and control of HBV. Furthermore, the meaningful mutants may be used for improving diagnostic assays and treatment or designing new vaccines for HBV.

Acknowledgments. This review is part of the work supported by Grants from the National Natural Science Foundation of China (8157081287).

\section{References}

Bauer T, Weinberger K, Jilg W (2002): Variants of Two Major T Cell Epitopes Within the Hepatitis B Surface Antigen Are Not Recognized by Specific T Helper Cells of Vaccinated Individuals. Hepatology 35, 455-465. https://doi. org/10.1053/jhep.2002.30903

Beasley RP (2009): Rocks along the road to the control of HBV and HCC. Ann. Epidemiol. 19, 231-234. https://doi. org/10.1016/j.annepidem.2009.01.017

Chang MH (2010): Breakthrough HBV infection in vaccinated children in Taiwan: surveillance for HBV mutants. Antivir. Ther. 15, 463-469. https://doi.org/10.3851/IMP1555

Chotiyaputta W, Lok AS (2009): Hepatitis B virus variants. Nat. Rev. Gastroenterol. Hepatol. 6, 453-462. https://doi. org/10.1038/nrgastro.2009.107

Cooreman MP, Leroux-Roels G, Paulijb WP (2001): Vaccine- and Hepatitis B Immune Globulin-Induced Escape Mutations of Hepatitis B Virus Surface Antigen. J. Biomed. Sci. 8, 237-247. https://doi.org/10.1007/BF02256597

Edmunds WJ, Medley GF, Nokes DJ, O'Callaghan CJ, Whittle HC (1996): Epidemiological patterns of hepatitis $\mathrm{B}$ virus $(\mathrm{HBV})$ in highly endemic areas. Epidemiol. Infect. 117, 313-325. https://doi.org/10.1017/ $\underline{\text { S0950268800001497 }}$

FitzSimons D, Hendrickx G, Vorsters A, Van Damme P (2013): Hepatitis B vaccination: A completed schedule enough to control HBV lifelong? Vaccine 31, 584-590. https:// doi.org/10.1016/j.vaccine.2012.10.101

Franzese O, Kennedy PT, Gehring AJ, Gotto J, Williams R, Maini MK (2005): Modulation of the CD8+-T-cell response by CD4+ CD25+ regulatory T cells in patients with hepatitis B virus infection. J. Virol. 79, 3322-3328. https://doi. org/10.1128/JVI.79.6.3322-3328.2005

Gerlich WH (2006): Breakthrough of hepatitis B virus escape mutants after vaccination and virus reactivation. J. Clin. Virol. 36, S18-22. https://doi.org/10.1016/S1386-6532(06)80004-1

Honorati MC, Dolzani P, Mariani E, Piacentini A, Lisignoli G, Ferrari C (1997): Epitope specificity of Th0/Th2 CD4+ Tlymphocyte cloones induced by vaccination with $\mathrm{rHBs} \mathrm{Ag}$ vaccine. Gastroenterology 112, 2017-2027. https://doi. org/10.1053/gast.1997.v112.pm9178695

Hudu SA1, Harmal NS, Saeed MI, Alshrari AS, Malik YA, Niazlin MT (2015): Naturally occurring hepatitis B virus surface antigen mutant variants in Malaysian blood donors and vaccinees. Eur. J. Clin. Microbiol. Infect. Dis. 34, 13491359. https://doi.org/10.1007/s10096-015-2358-1
Hsu HY, Chang MH, Ni YH (1997): Surface gene mutants of hepatitis B virus in infants who develop acute or chronic infection despite immunoprophylaxis. Hepatology 26, 786-791. https://doi.org/10.1002/hep.510260336

Hsu HY, Chang MH, Ni YH, Chen HL (2004): Survey of hepatitis B surface variant infection in children 15 years after nationwide vaccination program in Taiwan. Gut 53, 1499-1503. https://doi.org/10.1136/gut.2003.034223

Hsu HY, Chang MH, Ni YH, Chiang CL, Wu JF, Chen HL (2015): Universal infant immunization and occult hepatitis B virus infection in children and adolescents: a populationbased study. Hepatology 61, 1183-1191. https://doi. org/10.1002/hep. 27650

Kfoury Baz EM, Zheng J, Mazuruk K, Van Le A, Peterson DL (2001): Characterization of a novel hepatitis B virus mutant: demonstration of mutationinduced hepatitis $B$ virus surface antigen group specific 'a' determinant conformation change and its application in diagnostic assays. Transfus. Med. 11, 355-362. https://doi.org/10.1046/j.1365-3148 2001.00323.x

Larralde O, Dow B, Jarvis L, Davidson F, Petrik J (2013): Hepatitis B escape mutants in Scottish blood donors. Med. Microbiol. Immunol. 202, 207-214. https://doi.org/10.1007/ s00430-012-0283-9

Lai MW, Lin TY, Tsao KC (2012): Increased seroprevalence of HBV DNA with mutations in the sene among individuals greater than 18 years old after complete vaccination. Gastroenterology 143, 400-407. https://doi.org/10.1053/j. gastro.2012.05.002

Li R, Yang J, Gong J, Li Y, Huang Z, Fang K (2004): Efficacy of hepatitis $B$ vaccination on hepatitis $B$ prevention and on hepatocellular carcinoma. Chin. J. Epidemiol. 25, 385-387.

Lin YM, Jow GM, Mu SC, Chen BF (2013): Naturally Occurring Hepatitis B Virus B-Cell and T-Cell Epitope Mutants in Hepatitis B Vaccinated Children. Sci. Wrld. J. 1, 571875. https://doi.org/10.1155/2013/571875

Luongo M, Critelli R, Grottola A, Gitto S, Bernabucci V, Bevini M (2015): Acute hepatitis B caused by a vaccine-escape HBV strain in vaccinated subject: Sequence analysis and therapeutic strategy. J. Clin. Virol. 62, 89-91. https://doi. org/10.1016/j.jcv.2014.11.029

Ma Q, Wang Y (2012): Comprehensive analysis of the prevalence of hepatitis B virus escape mutations in the major hydrophilic region of surface antigen. J. Med. Virol. 84, 198-206. https://doi.org/10.1002/jmv.23183

Mancini-Bourgine M, Fontaine H, Brechot C, Pol S, Michel ML (2006): Immunogenicity of a hepatitis B DNA vaccine administered to chronic HBV carriers. Vaccine 24, 44824489. https://doi.org/10.1016/j.vaccine.2005.08.013

Mast EE, Weinbaum CM, Fiore AE, Alter MJ, Bell BP, Finelli L et al. (2006): A comprehensive immunization strategy to eliminate transmission of hepatitis B virus infection in the United States. Recommendations of the Advisory Committee on immunization practices (ACIP) Part II: immunization of adults. MMWR Recomm. Rep. 55 (RR-16), 1-33. 
Mendy M, D'Mello F, Kanellos T, Oliver S, Whittle H, Howard CR (2008): Envelope protein variability among HB Infecte asymptomatic carriers and immunized children with breakthrough infections. J. Med. Virol. 80, 1537-1546. https://doi.org/10.1002/jmv.21221

Michler T, Russo C, Zhang K (2014): Correlation of fulminant hepatitis $\mathrm{B}, \mathrm{HBeAg}$ start-codon depletions and escape mutations in the 2nd loop of the HBsAg a-determinant. Int. J. Infect. Dis. 21, 312. https://doi.org/10.1016/j. ijid.2014.03.1067

Mizukoshi E1, Sidney J, Livingston B, Ghany M, Hoofnagle JH, Sette A, Rehermann B (2004): Cellular immune responses to the hepatitis B virus polymerase. J. Immunol. 173, 58635871. https://doi.org/10.4049/jimmunol.173.9.5863

Schweitzer A, Horn J, Mikolajczyk RT, Krause G, Ott JJ (2015): Estimations of worldwide prevalence of chronic hepatitis B virus infection: a systematic review of data published between 1965 and 2013. Lancet 386, 1546-1555. https:// doi.org/10.1016/S0140-6736(15)61412-X

Schirmbeck R, Dikopoulos N, Kwissa M, Leithäuser F, Lamberth K, Buus S (2003): Breaking tolerance in hepatitis B surface antigen (HBsAg) transgenic mice by vaccination with cross-reactive, natural HBsAg variants. Eur. J. Immunol. 33, 3342-3352. https://doi.org/10.1002/eji.200324403

Shahmoradi S, Yahyapour Y, Mahmoodi M, Alavian SM, Fazeli Z, Jazayeri SM (2012): High prevalence of occult hepatitis B virus infection in children born to HBsAg-positive mothers despite prophylaxis with hepatitis $B$ vaccination and HBIG. J. Hepatol. 57, 515-521. https://doi.org/10.1016/j. ihep.2012.04.021

Shi L, Wang JM, Ren JP, Cheng YQ, Ying RS, Wu XY (2014): KLRG1 impairs CD4+T cell responses via p16ink4a and p27 kip1 pathways: role in hepatitis $B$ vaccine failure in individuals with hepatitis C virus infection. J. Immunol. 192, 649-657. https://doi.org/10.4049/jimmunol.1302069

Tai PC, Banik D, Lin GI, Pai S, Pai K, Lin MH (1997): Novel and frequent mutations of hepatitis B virus coincide with a major histocompatibility complex classI-restricted T-cell epitope of the surface antigen. J. Virol. 71, 4852-4856.

Valats JC, Tuaillon E, Funakoshi N, Hoa D, Brabet MC, Bolloré K (2010): Investigation of memory B cell responses to hepa- titis B surface antigen in health care workers considered as non-responders to vaccination. Vaccine 28, 6411-6416. https://doi.org/10.1016/j.vaccine.2010.07.058

Wen WH, Chen HL, Ni YH, Hsu HY, Kao JH, Hu FC (2011): Secular trend of the viral genotype distribution in children with chronic hepatitis B virus infection after universal infant immunization. Hepatology 53, 429-436. https://doi. org/10.1002/hep.24061

Wu C, Deng W, Deng L, Cao L, Qin B, Li S (2012): Amino Acid Substitutions at Positions 122 and 145 of Hepatitis B Virus Surface Antigen (HBs Ag) Determine the Antigenicity and Immunogenicity of $\mathrm{HBs} \mathrm{Ag}$ and Influence In Vivo HBs Ag Clearance. J. Virol. 86, 4658-4669. https://doi. org/10.1128/JVI.06353-11

Wu C, Zhang X, Tian Y, Song J, Yang D, Roggendorf M (2010): Biological significance of amino acid substitutions in hepatitis B surface antigen ( $\mathrm{HBsAg}$ ) for glycosylation, secretion, antigenicity and immunogenicity of HBsAg and hepatitis B virus replication. J. Gen. Virol. 91, 483-4492. https://doi.org/10.1099/vir.0.012740-0

Ye Q, Shang SQ, Li W (2015): A new vaccine escape mutant of hepatitis B virus causes occult infection. Hum. Vaccin. Immunother. 11, 407-410. https://doi.org/10.4161/216 45515.2014.994461

Ziaee M, Javanmard D, Sharifzadeh G, Hasan Namaei M, Azarkar G (2016): Genotyping and Mutation Pattern in the Overlapping MHR Region of HBV Isolates in Southern Khorasan, Eastern Iran. Hepat. Mon. 16, e37806. https:// doi.org/10.5812/hepatmon.37806

Zhang X, Kraft A, Broering R, Schlaak JF, Dittmer U, Lu M (2012): Preclinical development of TLR ligands as drugs for the treatment of chronic viral infections. Expert Opin. Drug. Discov. 7, 597-611. https://doi.org/10.1517/17460441. 2012.689281

Zhu CL, Liu P, Chen T, Ni Z, Lu LL, Huang F (2011): Presence of immune memory and immunity to hepatitis $B$ virus in adults after neonatal hepatitis B vaccination. Vaccine 29, 78357841. https://doi.org/10.1016/j.vaccine.2011.07.098

Zuckerman AJ (2000): Effect of hepatitis B virus mutants on efficacy of vaccination. Lancet 355, 1382-1384. https://doi. org/10.1016/S0140-6736(00)02132-2 